\title{
Die Studien zum Terrorismus
}

\section{Terrorismus als Gegenstand wissenschaftlicher Analyse}

Die Terrorismus forschung im Auftrag der Ständigen Konferenz der Innenminister der Länder war ein von Beginn an schwieriges Unternehmen. Mit Spannung und Mißtrauen wurde nach Bekanntwerden der Pläne zu einer diesbezüglichen Forschung beobachtet, wer sich wohl »dafür hergeben«würde, im Auftrag einer Institution, der eindeutige Interessen zuzuschreiben sind, das Problem Terrorismus wissenschaftlich zu analysieren. Was war zu erwarten von einem Forschungsprojekt, das bereits in seiner Anlage derart der Gefahr der Wahrnehmungsverkürzung ausgesetzt war? Zudem: Welche Motive veranlaßten die politische Administration, nun gerade bei den Sozialwissenschaftlern Auskunft über den Terrorismus zu verlangen?

Orientiert man sich an dem Vorwort des Herausgebers, dem Bundesministerium des Inneren, so sollte die wissenschaftliche Forschung »dazu beitragen, Wissenslücken über die Entstehung und Entwicklung des Terrorismus zu schließen, Vorurteile abzubauen und die öffentliche Diskussion zu versachlichen, um Fehler in der Einschätzung des Terrorismus und in der Reaktion auf ihn zu vermeiden.« (I/5)* Dies ist ein in doppelter Hinsicht problematischer Anspruch. Zum einen zeigt ein Blick auf die Geschichte des Terrorismus, daß je nach historischem Standpunkt unterschiedliche Analysen und Erklärungen möglich sind. Wie Laqueur in diesem $\mathrm{Zu}$ sammenhang anmerkt: »Terrorismus kann nur aufgrund seiner historischen Entwicklung, nicht durch Zahlen und Daten, die mehr oder weniger willkürlich in Computer gefüttert wurden, verstanden werden. «' Anders herumgewendet, eine Analyse, die diese historische Perspektive nicht berücksichtigt, bleibt ihren eigenen Voraussetzungen gegenüber blind. Zum anderen: Spezielle Probleme lauern in der Analyse des Terrorismus für den Analytiker selbst. "Sozialforscher, die oft gegenüber halboffiziellen oder Regierungsstellen in der Verantwortung stehen, deren Hauptinteresse ökonomische und politische Ruhe ist, können ihre Modelle leicht nach den Wünschen ihrer Klienten maßschneidern und dabei viele Möglichkeiten, Terrorismus anders zu analysieren, übersehen. ${ }^{2}$

Bedenkt man die immer noch vorhandene politische Brisanz des Themas "Terrorismus", so wird deutlich, daß es sich hier nicht um irgendein staatlich initiiertes Forschungsprojekt handelt, bei dem wissenschaftliche Experten lediglich ihre Sachkompetenz in den Dienst praktischer Interessen stellen. Einerseits liegt in der Idee zu einer breit angelegten Auseinandersetzung mit dem Terrorismus, außerhalb der praktischen Zwänge der mit ihm beschäftigten Institutionen, die faszinierende Chance, im Medium der (Sozial-) Wissenschaft einen gesellschaftlichen Reflexionsprozeß zu inszenieren, der Gesellschaft eine vernünftige Auseinandersetzung mit einem zentralen Aspekt der eigenen politischen Geschichte der letzten zwanzig Jahre

\footnotetext{
* Die erste Zahl in Klammern bezieht sich auf den jeweiligen Band: Band I I. Fetscher u. a., Ideologien und Strategien, Opladen 1981; Band 2 H. Jäger u. a., Lebenslaufanalysen, Opladen 1981; Band 3 W. von Baeyer-Katte u. a., Gruppenprozesse, Opladen 1982. Die zweite Zahl in Klammern gibt die jeweilige Seite an.

W. Lacueur, Terrorismus, Kronberg/Taunus 1977, S. 214

2 I. L. Horowitz, Transnational Terrorism, Civil Liberties and Social Science, in: Y. Alexander, S. M Finger (eds.) Terrorism: Interdisciplinary Perspectives, New York 1977, S. 284 , übersetzt vom Verfasser.
} 
zu ermöglichen. Andererseits besteht die Gefahr, daß die Wissenschaft hier unter die Räder politischer Opportunität gerät und die möglichen Ansätze zur Einleitung einer rationalen Diskussion über Terrorismus zwischen bürokratischen Hemmnissen, Ausgewogenheitsüberlegungen und partikularen Interessen zerrieben werden.

Die im politischen Terrorismus angelegte radikale Herausforderung des Staates entzieht auch jeder Debatte über Terrorismus schnell das sichere Fundament. Insbesondere für die Vertreter derjenigen Theorien, aus deren Repertoire, wie verzerrt auch immer, sich die terroristische Szene ihre Ideologie zimmerte, entsteht der Zwang zur Verteidigung: »Läßt man sich aber einmal in diese Rolle drängen, so hat man sich damit bereits auf den Boden des neuerdings um sich greifenden Gesinnungsstrafrechts gestellt, auf dem die Angeklagten immer die Verlierer sind, ganz gleich, ob es ihnen nun gelingt, ihre Unschuld zu bewiesen oder nicht: Sie haben sich nämlich darauf eingelassen, einen Harmlosigkeitsbeweis für ihre Gedanken zu führen, und hierin allein steckt schon ein Stück Selbstverpflichtung, in Zukunft nur noch harmlose Gedanken zu haben. ${ }^{3}$ Die Warnung vor falschen Distanzierungen und Solidarisierungen und die Forderung nach einem rationalen Verhältnis zum Terrorismus ${ }^{4}$ sind in diesem Zusammenhang berechtigt, wie ihnen nachzukommen sei, ist jedoch eine andere Frage. Es ist anzunehmen, daß den meisten Beteiligten an diesem Forschungsprojekt diese Problematik von vornherein klar war. Möglicherweise war es im einen oder anderen Fall auch ein ausschlaggebendes Motiv, sich an diesem Projekt zu beteiligen, um die Chance der rationalen Auseinandersetzung mit dem Terrorismus nicht nur der »Gegenseite « zu überlassen. Denn es ist bekannt, daß auch die Wissenschaft ihr Süppchen über den Terrorismus kocht: Läßt sich für die einen über das Scharnier der Schreibtischtäterthese der Terrorismus als Kronzeuge für die fatalen Folgen linker Gesellschaftstheorie stilisieren, so fühlen sich, wie Wellmer feststellt, die anderen aufgerufen nachzuweisen, daß dieser Zusammenhang nicht existiert. Auf dem Forum der öffentlichen Diskussion schlägt sich diese Situation zunächst nieder als Feldvorteil des konservativen Lagers. Die historische Entwicklung, die dazu geführt hat, daß der Terrorismus objektiv der Reaktion in die Hände arbeitet, bringt die Linke in Bedrängnis. Gerade auch unter diesem Aspekt hätten die Analysen zum Terrorismus die Möglichkeit bieten können, die Debatte zu versachlichen und Terrorismus zu reflektieren als Ausdruck von Systempathologien und der Preisgabe linker Moral und Theorie, die Eskalation der Gewalt nachzuzeichnen als einen Aufschaukelungsprozeß von terroristischen Akten und staatlicher Reaktion.

Insgesamt ist es jedoch nicht gelungen, dies zu leisten. Dies hat meines Erachtens mehrere Ursachen. Zunächst ist hier die Gesamtkonzeption der Studie zu nennen, insbesondere die entschärfende Aufteilung der Forschung in die Teilaspekte "Ideologien und Strategien " (Band 1), "Lebenslaufanalysen " (Band 2), "Gruppenprozesse (Band 3) und "Gesellschaftliche Prozesse und Reaktionen« (Band 4). Die Konzentration der Forschung auf jeweils nur einen dieser Aspekte, die von den meisten Autoren auch durchgehalten wird, verhindert genau das, was eigentlich hätte das Ziel eines solchen Forschungsprojekts sein müssen, die umfassende Auseinandersetzung mit dem Gegenstand als realem Phänomen und nicht als teildisziplinär zu analysierendem Datenhaufen. Von besonderem Interesse ist dabei die Tatsache, daß der Band ${ }_{4}$, der von der Themenstellung wohl noch die umfassendste

\footnotetext{
3 A. Wellmer, Terrorismus und Gesellschaftskritik, in: J. Habermas (Hg.), Stichworte zur geistigen Situation der Zeit, Frankfurt/Main I979, S. 26 sff.

4 A. a. O., S. 267.
} 
Perspektive verspricht, bis jetzt noch nicht erschienen ist, obwohl Querverweise auf den entsprechenden Forschungsbericht in den anderen Beiträgen enthalten sind. Leider ist über die Gründe für diese Verzögerung nichts bekannt.' Möglicherweise handelt sich hier um einen unintendierten Nebeneffekt einer Proporz- und Ausgewogenheitsstrategie, die das Spektrum politischer Positionen in der bundesdeutschen Geistes- und Sozialwissenschaft im Rahmen des Berechenbaren vertreten sehen wollte. Daß man sich durch die Beteiligung von methodisch informierten und theoretisch versierten Linken ein Kuckucksei ins ausgewogene Nest legte, war für die Herausgeber vielleicht nicht abzusehen.

Der zweite Grund liegt in der Reglementierung des Forschungsprozesses durch die Administration. Wissenschaft ist prinzipiell öffentlich. Wenn aus Gründen der Staatsräson diese Öffentlichkeit nicht hergestellt werden kann, dann verliert die wissenschaftliche Arbeit einen wesentlichen Teil ihrer Legitimation. Dies gilt nicht nur für die Verzögerung der Publikation des 4. Bandes, sondern ebenfalls für die mit Geheimhaltungsinteressen begründete Art des Umgangs mit Daten in den empirischen Projekten der veröffentlichten drei Bände. Erlebnisberichte aus dem verbotenen Reich des Terrorismus, bzw. genauer aus dem Bereich des staatlich erfaßten Terrorismus, aus dem nicht ausgeführt werden darf, was aus der Sicht der Instanzen geheim zu bleiben hat, haben einen geringen wissenschaftlichen Wert. Ziel empirischer Forschung ist es, unbekannte Zusammenhänge anhand der Analyse und Interpretation von Daten aufzuklären. Adressat und Prüfstein der Ergebnisse ist die Leserschaft der veröffentlichten Ergebnisse. Wie soll diesen allgemeinen und trivialen Ansprüchen genügt werden, wenn über die Zusammenhänge, aus denen die Daten entnommen sind, nicht geredet werden darf? Die Wissenschaftlichkeit kommt bei einigen Beiträgen dieser Bände eher im wallenden Gewand des Alchemisten als im weißen Kittel des neuzeitlichen Forschers daher. Manche Autoren ziehen sich auf einen naiven Standpunkt zurück und destillieren aus fragwürdigen Zahlen fragwürdige Interpretationen. Wieder andere lassen ihren in systemtheoretischem Jargon gefaßten Gedanken freien Lauf bei der Abfassung ihrer Beiträge, über deren zugrundeliegende Datenbasis sie den Leser weitgehend im unklaren lassen. Bemerkenswert ist dabei, wie der Hinweis auf die aktuelle Bedeutung des Themas die rigiden wissenschaftlichen Standards ersetzt. Heiligt die Aktualität den methodischen Schlendrian? Was ist das für eine Art "wissenschaftliche Auseinandersetzung mit dem Problem Terrorismus «, bei der ein Forscher auf die Frage: Welche Belege haben Sie für diese These? antwortet: Das darf ich nicht sagen? Daß »die Pflicht zur vertraulichen Behandlung des vorgefundenen Materials durch besondere Verpflichtungserklärung dem Strafrecht unterstellt wurde« (2/137), erinnert in fataler Weise an die Behandlung strategisch relevanter Forschung im Kriegsfall.

An der Problematik der Datenbasis läßt sich die eigentümliche Allianz von staatlichen Interessen und wissenschaftlichen Methoden der Untersuchung bei der Terrorismusforschung zeigen. Die den meisten Untersuchungen zugrundeliegende Population $(n=250)$ wird definiert über strafrechtliche Kriterien. »Die Datenerhebung erstreckte sich auf Personen, die bis zum Ende des Jahres 1978 entweder wegen eines Vergehens gegen den $\$$ i 29a StGB mit Haftbefehl gesucht, angeklagt oder verurteilt worden waren oder (nach dem Inhalt des gegen sie ergangenen Urteils) verurteilt worden wären, wenn zu diesem Zeitpunkt \I29a StGB bereits in Kraft gewesen

\footnotetext{
5 Aufschlußreich ist in diesem Zusammenhang allerdings die Art, wie ein Autor des vierten Bandes, Fritz Sack, seinen Beitrag an anderer Stelle zitiert: "Die Rolle staatlichen und gesellschaftlichen Handelns bei der Genese gewaltförmiger Auseinandersetzungen und terroristischer Konflikte, unv. Ms., erscheint (hoffentlich demnächst) in Opladen, Westdeutscher Verlag«, F. Sack, in: Kriminologisches Journal 1982 , S. 253 .
} 
wäre." (2/19) In dieser Population sind 23 Angehörige rechtsextremistischer Gruppen enthalten, die aus kosmetischen Gründen durch die Analyse mitgeschleppt werden. Die normative Setzung, daß eine Person Terrorist ist, manifestiert in einer (möglichen) rechtskräftigen Verurteilung, ersetzt hier wissenschaftliche Standards der Definition von Untersuchungspopulationen. Gerechtfertigt, d. h. für die eigene Analyse folgenreich diskutiert wird dieser Sachverhalt an keiner Stelle. Verwiesen wird lediglich auf die allgemeine Datenproblematik. Wird aber diese justiziell erzeugte Population post-hoc sozialwissenschaftlich untersucht, ohne die Konstitutionsbedingungen dieser Population zu berücksichtigen, sowie unter Beschränkung auf Fragestellungen, die den juristischen Definitionskontext ausblenden, so werden dadurch sozialwissenschaftliche Aussagen über eine anhand normativer Kriterien erzeugte Gruppe mit wissenschaftlichen Methoden gerechtfertigt. Dadurch entsteht dann der (gewünschte?) Eindruck, die juristische Bearbeitung des Problems korrespondiere in ihren Ergebnissen einer sozialwissenschaftlich abgesicherten Homogenität oder Struktur dieser Gruppe.

Im folgenden sollen die drei bisher veröffentlichten Bände der Analysen zum Terrorismus kurz vorgestellt werden. Eine detaillierte immanente Auseinandersetzung mit allen darin enthaltenen Beiträgen läge jenseits der Kompetenz des Autors und außerhalb des Rahmens dieser Arbeit. Es soll daher versucht werden, ohne Anspruch auf Vollständigkeit, bruchstückhaft und exemplarisch zu den Forschungsergebnissen Stellung zu nehmen. Die leitende Idee ist dabei, daß es sich hier nicht nur um die Präsentation wissenschaftlicher Ergebnisse handelt, sondern um einen von Wissenschaftlern geleisteten Beitrag zu einem gesellschaftlich kontrovers diskutierten Thema, der nicht nur jeweils immanent methodisch oder theorieorientiert kritisierbar ist, sondern in einem weiteren Kontext kritisch gesehen werden muß: Als staatlich angeforderter und öffentlich präsentierter Beitrag der Sozialwissenschaft zur Terrorismusdebatte.

\section{Band 1: Ideologien und Strategien \\ Da haben Sie die Marxisten aber falsch verstanden - dann hätten Sie anders schreiben sollen: Fetscher und Rohrmoser über Ideologien und Strategien der Terroristen}

Der erste Band der Analysen zum Terrorismus mit dem Titel »Ideologien und Strategien« enthält zwei Beiträge: "Ideologie der Terroristen in der Bundesrepublik Deutschland " von Iring Fetscher, Herfried Münkler und Hannelore Ludwig und "Ideologische Ursachen des Terrorismus "von Günter Rohrmoser unter Mitarbeit von Jörg Fröhlich. Fetscher und Rohrmoser haben, am Ende des Bandes abgedruckt, jeweils noch ein Nachwort verfaßt, in dem sie auf den Beitrag des anderen kurz eingehen. Die Methode, die Fetscher u. a. ihrer Analyse zugrundelegen, wird als immanent, Rohrmosers Vorgehen als transzendent beschrieben. Fetscher zieht als Datenmaterial heran

„das gesamte gedruckte und vervielfältigte Material, das von Angehörigen der RAF, der Bewegung 2. Juni und der Revolutionären Zellen veröffentlicht wurde, und darüber hinaus... Zellenzirkulare, soweit sie ideologisch relevante Aussagen enthielten." (1/16)

Das Material wurde chronologisch geordnet, um die Entstehung von Argumentationslinien nachzuweisen. Leitend war bei der Vorgehensweise die Feststellung,

"daß Ideologien zwar eine wichtige Rolle für die Formulierung des Selbstverständnisses der Angehörigen dieser Gruppe bildeten, daß sie aber nicht oder doch nicht die ausschlaggebenden Motive für den Übergang zum bewaffneten Kampf waren." (1/16) 
Hier zeigt sich bereits ein zentrales Problem: Wie soll, ohne Einbezug der den anderen Experten zugeteilten Problemdimensionen, eine Entscheidung über die Bedeutung von Ideologien (als Handlungsmotiv) getroffen werden? Natürlich, so Fetscher, müssen für eine endgültige Antwort die Ergebnisse der anderen Teilprojekte noch abgewartet werden. Möglicherweise lassen sich dort noch andere Motive finden.

Der Weg den Fetscher u. a. in ihrer Analyse einschlagen, soll die "Grenze rationalen Verstehens - ohne Rückgriff auf psychologische Hypothesen - soweit wie irgend möglich hinausschieben. «(I/33) Das wesentliche Verdienst von Fetscher und seinen Mitarbeitern ist die Aufbereitung des Denkwegs der prominenten Vertreter der bundesdeutschen Terrorszene. Deutlich treten dabei die Unterschiede zwischen den führenden Köpfen, insbesondere zwischen Horst Mahler und Ulrike Meinhof in den Vordergrund. Es wird gezeigt, wie beide auf ihre je eigene Art versuchen, durch den Umbau ihres ideologisch-theoretischen Bezugsrahmens ihr Handeln und dessen offensichtlichen Mißerfolg mit ihren theoretischen Analysen zur Deckung zu bringen. Die verzweifelte Suche nach dem revolutionären Subjekt, die vom »Industrieproletariat" bis zur "mythisierten Automation « (1/83 ff.) führt und deren Mißerfolg schließlich die Motivation für terroristische Aktionen auf die Selbsterhaltung und Befreiung von Genossen zusammenschrumpfen läßt, wird anhand des verfügbaren schriftlichen Materials klar dargestellt. Der zweite Hauptteil des Beitrags von Fetscher u. a. geht der Bedeutung zweier zentraler Begriffe der terroristischen Ideologie - Faschismus und Imperialismus - nach. Auch hier der sicher geführte Nachweis, daß die Verwendung dieser Begriffe durch die Terroristen einer kritischen Überprüfung nicht standhält.

Am Ende ist klar, daß aus der linken Theorietradition keine handlungsleitenden Motive der Terroristen abgeleitet werden können. Der in den RAF-Schriften auffindbare Bezug zu diesen Theorien ist gekennzeichnet durch Verkürzungen, Mißinterpretationen und Verzerrungen. So weit, so gut. Nur, was ist damit gewonnen? Die Schreibtischtäterthese ist widerlegt worden auf der Ebene des akademischen Diskurses, der platte Vorwurf, terroristische Gewaltakte seien in einer klar nachkonstruierbaren Kausalkette aus der Rezeption (neo-)marxistischer Theorien ableitbar, ist entkräftet. Das Phänomen Terrorismus ist dadurch nicht verständlicher geworden. Es hat den Anschein, daß Fetscher u. a. zu sehr auf dem Nebenkriegsschauplatz der ideologischen Diffamierungskampagne, der sich die Linke hier ausgesetzt sieht, gekämpft haben. Der Durchbruch zu einer Analyse des Terrorismus als Element historischer Entwicklungen - jenseits der zu Recht kritisierten Psychologisierung des Phänomens - ist nicht gelungen. Dies liegt sicher, wie bereits erwähnt, an der vorgegebenen inhaltlichen Beschränkung auf Ideologien und Stragegien. Andererseits wäre aber auch in diesem Rahmen mehr möglich gewesen, ein konkreterer Bezug auf jene Systempathologien, die Terrorismus hervorbringen. Statt dessen wird hier immer von der zwar wichtigen, in diesem Zusammenhang aber wenig hilfreichen Position aus argumentiert, daß Terrorismus kein Instrument fortschrittlicher Politik in der BRD sein könne und daß die »konsequente Auflösung des Widerspruchs (im Denken der Terroristen, R. K.) zur Durchbrechung der imaginierten Realität hätte führen müssen und damit womöglich eine Abkehr vom Terrorismus bewirkt hätte. « ( $1 / 82$ ) Durch die Vernachlässigung der realen gesellschaftlichen Verhältnisse bei der Analyse gelangt Fetscher durch die Hintertür dann doch wieder zu einer psychologischen Erklärung:

"Diese offensichtliche Unfähigkeit, das eigene Handeln an der Realität zu orientieren und zu korrigieren, war uns ein Indiz dafür, daß hinter den theoretischen Erklärungen (der Terroristen, R. K.) Motive stecken müßten, die eher von Sozialpsychologen als vom Standpunkt der Theoriegeschichte aus zu lösen wären.« (I/341) 
Fetschers als immanent bezeichnetes Vorgehen greift zu kurz, da die hermeneutische Analyse den Rahmen des quasi einsam handelnden Individuums nicht verläßt. Gerade dieser Schritt aber wäre notwendig gewesen, um die Verschränkung von individuellen Denkwegen und den objektiven Verhältnissen, die sich in ihnen niederschlagen, zu begreifen. Die bizarre Logik terroristischen Handelns unter dem Aspekt zunehmenden Realitätsverlusts zu beschreiben, wie es hier geschieht, vernachlässigt das objektive Moment dieses Prozesses. Fetscher begründet diese Beschränkung in seinem Schlußwort wie folgt:

„Es gibt meines Erachtens sowohl objektive soziale und politische Ursachen für radikale oppositionelle Bewegungen ... als auch kulturelle, wie sie Rohrmoser in seiner Studie angesprochen hat. Wie beide zusammenhängen, das zu untersuchen wäre vielleicht eine weitere Aufgabe. Als Mitarbeiter an einem arbeitsteiligen Gesamtprojekt fühlte ich mich nicht legitimiert, sie zu übernehmen. " $(\mathrm{I} / 343)$

Die Chance, durch eine Analyse terroristischer Ideologie zu gehaltvollen Aussagen über den Zustand der bundesdeutschen Gesellschaft zu gelangen, wurde im Beitrag von Fetscher u. a. verspielt: Die einseitige, wenn auch zutreffende Betonung von Rezeptionsfehlern bei der Analyse der terroristischen Ideologie läßt den Aspekt der "gesellschaftlichen Produktion" von Terrorismus außen vor.

Nach der Lektüre von Fetschers Beitrag ist zunächst Rohrmosers mit einer gewissen Ironie vorgebrachte Einschätzung einleuchtend:

"Es ist die sehr plausible Konsequenz einer sogenannten immanenten Interpretation der Texte des Terrorismus, ihres ideologischen Gehalts, ihrer politischen Motive und ihrer theoretischen Rechtfertigung, daß die Frage an die Sozialpsychologie zur weiteren Bearbeitung als gegeben werden muß.« $(1 / 276)$

Für Rohrmoser ist die Frage, die Fetscher u. a. sich stellen, eine, »die nur beantworten kann, der ein Monopol auf die authentische Interpretation der Lehre marxistischer Klassiker kennt und diese anerkennt. Es ist ein innermarxistischer Streit, der für die Erklärung des Terrorismus und seiner Genese nur von untergeordneter Bedeutung ist. « ( $\mathrm{I} / 279)$. Die Art, in der Rohrmoser seinen Beitrag aufzieht, erinnert an die neokonservative Variante der Kritik politischer Kultur. Mangelnde Legitimität des Staates, die fehlende integrative Kraft liberaler Parteiendemokratie, Zerstörung des gesellschaftlichen Konsens sind für ihn die Ansatzpunkte seiner Darstellung des Terrorismus. Mit Offe wäre Rohrmosers Analyse unter die Kategorie derjenigen neokonservativen Ansätze einzuordnen, die das Versagen "präpolitische(r) kulturelle(r) Disziplinierungsleistungen $\aleph^{6}$ zur Ursache verschiedener pathologischer Entwicklungen stilisieren. Entsprechend ist für Rohrmoser der Terrorismus willkommener Anlaß, kulturkritisch die Pathologie des Systems zu beschwören. In gewisser Weise nutzt er unter neokonservativen Vorzeichen die Chance, die Fetscher u. a. vergeben haben. Er versucht, das Phänomen Terrorismus in »seinen« Kontext zu stellen:

»Es handelt sich ja beim deutschen Terrorismus nicht um ein pathologisches Randphänomen, als das es von vielen Bürgern empfunden wird, sondern um ein Symptom eines universalen Phänomens, das seine Evidenz in einer Welt gewinnt, in der in einem radikalen Sinn strittig ist, was die Prinzipien rationalpragmatischer Selbsterhaltung gebieten, ja in der strittig ist, was eigentlich verdient, für die Wirklichkeit gehalten zu werden. Ein ideologieunverdächtiger Standpunkt ist auch in der Beurteilung des politischen Terrorismus prinzipiell unmöglich. Die geistig-politische Auseinandersetzung mit dem Terrorismus, zu der hier ein Beitrag erwartet wird, ist schwieriger als es zunächst den Anschein hat." ( $(\mathrm{I} / 278)$

Mit dieser Bestimmung legt Rohrmoser die Ebene fest, auf der er zu argumentieren

6 C. Offe, Unregierbarkeit. Zur Renaissance konservativer Krisentheorien, in: J. Habermas, a. a. O., S. 310. 
gedenkt. Was er im folgenden in einem zeitdiagnostischen Rundschlag entwickelt, ist eine vornehme Variante der Schreibtischtäterthese. Seiner Meinung nach ist etwa die Trennung von Legalität und Legitimität des Staates im öffentlichen Bewußtsein eine der kritischen Theorie zurechenbare Entwicklung (vgl. I/287). Die Subversion der Frankfurter Schule ist für Rohrmoser eine ex negativo: nicht daß er ihre Analyse für falsch hielte - ihre Praxisverweigerung, die nicht geleistete konstruktive Wende kreidet er ihr an. Den Bogen von Lukács über Marcuse zu Sartre spannend, entwickelt Rohrmoser sein Schreckensgemälde vom nicht mehr legitimierbaren Staat, um dann, dem "geläuterten « Horst Mahler nicht unähnlich, auf Hegel rekurrierend, den Staat als Bewahrer und Garant der Sittlichkeit zu beschwören.

Die Vorgehensweise, ideengeschichtlich eine Genealogie des Terrorismus zu entwickeln, wobei alles, was linken Rang und Namen hat, in der Ahnenreihe der RAF untergebracht wird, entlarvt sich bereits beim ersten Lesen als Konstruktion, die weniger auf Erkenntnis abzielt als darauf, die Schwarzen Listen über die Roten vollständig zu zitieren. Es ist eine eigenartige Verkehrung der Verhältnisse, wenn Rohrmoser auf dem gesellschaftlichen Bezug terroristischen Handelns insistiert, um seine konservative Gesellschaftskritik damit zu transportieren, während Fetscher u. a. zu diesem Thema sich so gut wie nicht äußern.

Der erste Band der Studien zum Terrorismus ist insofern von Interesse, als er die Kontroverse über den Anteil gesellschaftskritischer Theorien an der Entstehung des Terrorismus ziemlich genau widerspiegelt. Die »Linke», vertreten durch Fetscher, operiert aus der Defensive, verteidigt akribisch, doch theoretisch allzu begrenzt die Reinheit der Lehre gegen die Beschmutzung durch eine vermeintliche Praxis; die »Rechte« nutzt die Gelegenheit, ihr inhaltlich nicht konkretisiertes Programm der geistig-moralischen Erneuerung anzubieten. So haben also die A-Länder und die B-Länder ihre Sichtweise zum Problem der Ideologie des Terrorismus zwischen zwei Buchdeckel gebracht.

\section{Band 2: Lebenslaufanalysen}

Auch dieser Band der Analysen zum Terrorismus ist zweigeteilt. Diesmal allerdings nicht so sehr ideologisch als eher methodisch. Den ersten Teil bilden die Analysen von Gerhard Schmidtchen, "Terroristische Karrieren «, und Lieselotte Süllwold, »Situationen in der Entwicklung von Terroristen«. Beide subsumierbar unter die $\mathrm{Ka}_{\text {- }}$ tegorie quantitativer Forschung. Der zweite Teil besteht aus qualitativen Studien mit psychoanalytischem Hintergrund. »Die individuelle Dimension terroristischen Handelns " von Herbert Jäger und "Die Entwicklung zu terroristischem Handeln als psychosozialer Prozeß« von Lorenz Böllinger.

\section{Die Astroshow - oder: Empirie ohne Daten}

Die ganze Armseligkeit (der staatlich verordneten wissenschaftlichen Aufbereitung) des empirischen Datenmaterials zur Terrorismusproblematik tritt dem Leser in Schmidtchens Beitrag über terroristische Karrieren gegenüber. Wenn man keine Daten hat, dann sollte man sie auch nicht analysieren. Die Beherrschung der Prozentrechnung und die Fähigkeit, Zahlen in geraden Reihen untereinanderzuschreiben, reichen als Handwerkszeug für »soziologische Analysen anhand von Fahndungsunterlagen und Prozeßakten«, so der Untertitel von Schmidtchens Beitrag, nicht aus. Schmidtchen scheint bei seiner Arbeit methodisch auch nicht ganz wohl 
gewesen zu sein. Zu Beginn finden sich bei ihm Hinweise auf die Beschränkungen, denen die Analyse aufgrund der Datenlage unterliegt, die dann allerdings folgenlos bleiben, denn danach wird hemmungs- und konzeptionslos prozentuiert und schlußgefolgert. Die methodische Genauigkeit beschränkt sich dabei auf die arithmetisch fehlerfreie Aufsummierung, so daß in der letzten Zeile fast immer einhundert Prozent herauskommen. Die Analyse basiert auf 250 Fällen. Die Daten zu diesen Fällen wurden von Beamten des Bundesinnenministeriums und der Länderinnenverwaltungen mit Hilfe eines von Schmidtchen erarbeiteten Erhebungsbogens erhoben. Insgesamt gelangten dabei von einem »breit angelegten Erhebungsbogen « 92 Positionen in die statistische Auswertung. Ein nicht näher quantifizierter Teil dieser Ermittlungspositionen basiert auf "Gesamturteilen und Eindrücken ... z. B. vom Gesundheitszustand der in die Erhebung eingegangenen Personen, über Verhaltenstendenzen und politische Orientierung der Bezugspersonen « (2/20) aus der Sicht der die Erhebung durchführenden Beamten. Diese Daten werden qualifiziert als Wiedergabe der Eindrücke der Beamten aufgrund der Aktensituation. (2/20) Schmidtchen sieht den Umgang mit diesem Material "methodologisch mehr der Archäologie als einer rigorosen Entscheidungsstatistik verpflichtet. « (2/20) Ob Archäologen in dieser Nähe zu Kartenlegern arbeiten, ist mir nicht bekannt.

Sind die Daten zur Verfügung, so gilt es, einen theoretischen Bezugsrahmen zu entwickeln. Bei 92 Ermittlungspositionen sicher kein einfaches Unterfangen. $\mathrm{Zu}-$ dem: "Terrorist wird man nicht über Nacht.« (2/14) Auf knapp einer Seite entfaltet Schmidtchen eine »allgemeine Theorie der Strukturcndynamik von Biographien «, die auf einer paradigmatischen Veröffentlichung von Mihaly Csekszentmihaly und Olga V. Beattie basiert. Demnach entwickelt jede Person ein "Lebensthema«, an dessen Anfang existentieller Streß steht, der dann auf eine begriffliche Formel gebracht wird. Danach werden Lösungsmöglichkeiten ventiliert, um schließlich die Entscheidung für einen Lösungsweg zu finden. $\gg$ Die Anwendung dieses Interpretationsschemas auf Terroristenkarrieren ist mit Präzisierungen und der Einführung weiterer Annahmen möglich." (2/16) Wie etwa der folgenden:

"Frustrationen können oberhalb der physisch erfahrbaren Armutsgrenze als Phantasieprodukte bezeichnet werden. Damit soll die Bedeutung solcher Frustrationen nicht relativiert werden. Sie sind wesentliches Moment gesellschaftlicher und humanitärer Entwicklung zum Besseren. Damit (!) wird aber auch eine Erklärung möglich, wieso auch Kinder aus gutbürgerlichen Kreisen psychologische und soziale Frustrationen erleiden können, die jene Stärke haben, daß sie ein Lebensthema abgeben. Gerade in den gebildeten Schichten stehen die intellektuellen Mittel zur Verfügung, um Gefühle der Unlust und des Unbehagens als Problem identifizieren und formulieren zu können." $(2 / 17)$

Mit derartigen Erklärungen ausgerüstet, startet Schmidtchen seinen Durchgang durch die terroristischen Karrieren. Beginnend bei Daten zu Jahrgang und Geschlecht über Bildung und Beruf, wobei dann aus der Feststellung, daß Linksterroristen aus besonders bildungsbeflissenen Familien kommen und auf anspruchsvolle Bildungswege geschickt werden, wichtige Schlußfolgerungen gezogen werden: »Terroristen sind also (sic) Kinder überdurchschnittlich ehrgeiziger Eltern«. (2/26) Das kann ja nicht gutgehen!

"Die Bildungsanstrengungen scheitern zu einem beträchtlichen Teil. Die Ausbildung wird nicht abgeschlossen. Die Tatsache, daß akademische Studien nicht abgeschlossen werden, hängt indes nur zum Teil mit dem Anschluß an eine politische Gruppierung und dem späteren Untertauchen zusammen. Diese Alternative wurde höher bewertet als der Abschluß des Studiums. Aufschlußreich ist hier der Vergleich der Terroristen, die die Universität mit und ohne Abschluß besucht haben ... Die Studienabbrecher kommen hauptsächlich aus kulturwissenschaftlichen Fächern, also Soziologie, Psychologie und Sprachen, und der Weg in die Kommune führt in der Regel aus dem Studium heraus. Unter den Bafög-Beziehern hat fast niemand sein Studium beendet. " $(2 / 26)$ 
Man sollte die Bedeutung des Bafög als analytisch bedeutsame Variable in der Terrorismusforschung nicht unterschätzen; denn: »Bafög steht jetzt für eine gewisse Isolation vom Elternhaus, gleichsam ein Hinweis auf abgebrochene Kommunikation, aber die Studentenförderung ist natürlich nicht die Ursache.» (2/29). Gott sei Dank.

Was macht ein Terrorist in der Freizeit, was interessiert ihn, so privat:

"Soweit aus den Prozeßakten und den Angaben über Studienrichtungen hervorgehen kann, scheint das dominierende Interesse der Linksterroristen Gesellschaft und Psychologie zu sein. Dahinter mag das Motiv stehen, den Menschen in seiner Abhängigkeit verstehen zu wollen. Darin unterscheiden sie sich deutlich vom Rechtsterrorismus. Weitere Interessengebiete sind Literatur, Politik (hier zunächst nur als passives Interesse registriert, ohne Eigenaktivität), Philosophie, Religion, Naturwissenschaften, Technik, Musik; und es folgen schließlich Reisen, Trampen, Wirtschaft und passives Interesse am Sport... Das sind wohlgemerkt die in den Akten irgendwie (!) dokumentierten und von den Beamten so eingeordneten Interessen - also kein vollständiges Bild.« (2/37)

Immerhin ein buntes! Auch im persönlichen Umgang scheinen die Terroristen, sind sie erst einmal hinter Schloß und Riegel, sehr freundlich zu sein:

"Der Kommunikationsstil der Linksterroristen scheint nach indirekter Beurteilung gewinnend zu sein ... Es fällt auf, daß das Urteil über die Intelligenz mit dem Eindruck yom persönlichen Gespräch kovariiert, hier also im wesentlichen gebildet wurde." $(2 / 40)$

Ob Intelligenz vielleicht nach Aktenlage beurteilt werden kann? Möglicherweise hängt die den Linksterroristen attestierte hohe praktische Intelligenz auch mit deren Mobilität zusammen, denn wie Schmidtchen herausgefunden hat, im "Durchschnitt haben die Linksterroristen in I, 8 Orten gelebt. «(2/44). Außerdem nur in Großstädten. "Progressive Subkulturen waren dort nicht nur geduldet, sondern tonangebend. «(2/44) Man denke nur an die Düsseldorfer Hippie-Bewegung.

Aber auch der persönliche Einfluß der Bezugspersonen sollte nicht unterschätzt werden. Nach Analyse der Werte, die die Bezugspersonen auf der nach rechts hin offenen Beamtenskala erhalten haben, haben die "späteren Terroristen schon geraume Zeit nicht mehr in einem pluralistischen Diskussionskontext gelebt. « (2/45) Hinzu kommen

"auffällige Verhaltensweisen vor dem Anschluß an eine terroristische Gruppe ... Das Leben in Kommunen und Wohngemeinschaften, die Beschränkung des Kontakts auf bestimmte Gruppen, Aggressivität nach außen, gegenüber Autoritäten, die Aufgabe regelmäßiger Tätigkeit, der Übergang zu einer stereotypen ideologischen Sprache und die zunehmende Isolierung. Ferner sind Stimmungsschwankungen zu verzeichnen, stärkerer Wechsel der Beziehungspersonen, Aufgabe früherer Interessen und Hobbies, planloses Herumlungern, rasch wechselnde berufliche Ziele.« (2/48)

Nun kann bei Schmidtchen in Kapitel Io der Terrorist wie eine reife Pflaume vom Baum des Bösen fallen. Er hat letztlich »eine Struktur zum Gegner und Personen als Freunde."(2/55)

Auch der sich anschließende Beitrag von Süllwold, der auf den gleichen Daten basiert, widmet sich der Frage: Wie wird man Terrorist? Zusätzlich werden hier weitere Informationen über 65 Fälle ausgewertet; welche Informationen das sind, das wird dem Leser nicht mitgeteilt. Die zentrale theoretische Grundprämisse der Analyse von Süllwold ist die Auffassung,

"daß jede persönliche Entwicklung, auch die zum Terroristen, ein Wechselwirkungsprozeß zwischen verschiedenen Bedingungsfaktoren ist. Die entsprechenden Einstellungen, Affekte und letztlich Verhaltensweisen haben in jedem Fall eine Vorgeschichte, sie entstehen nicht über Nacht. $\lll(2 / 8 I)$ 
weiteren Verlauf der Darstellung sammeln sich auch hier die bekannten Eigenschaften von Terroristen. In Kommunen begeben sie sich, weil ihnen dies eine zeitliche Verzögerung des Erwachsenwerdens ermöglicht, weil dort ein zuvor unzureichender Sozialisationsprozeß nachgeholt werden kann. Auch das Vorhandensein von Kontaktstörungen, das übersteigerte Bedürfnis nach Beachtung, aufgelöste Partnerbeziehungen und mißlungene soziale Anpassung führen in die Kommune. Diese hat eine Auffangfunktion für Problemfälle.

Unter der Abteilung »Psychische Störungen« (Kap. 5) erfahren wir, daß es »den typischen Terroristen nicht gibt.« (2/103) Nach der Lektüre von Süllwold sollten wir uns eher einen Haufen marihuanarauchender, in Wohngemeinschaften wohnender Bettnässer mit abgebrochener Ausbildung und gestörtem Realitätsverhältnis vorstellen. Hier werden in der Tat die Wissenslücken über die Entstehung des Terrorismus geschlossen, wie dem Leser im Vorwort angekündigt wurde.

\section{Psychoanalyse und Terrorismus - Zwischen Rekonstruktion und subjektivem Gefühl}

Der Beitrag von Herbert Jäger $»$ Die individuelle Dimension terroristischen Handelns ist hier von Interesse, weil er einen sehr ausführlichen Teil über die allgemeinen Probleme der Terrorismusforschung sowie die konkreten Probleme bei der eigenen Projektarbeit enthält. Es ist vermutlich kein Zufall, daß dieser Problematik gerade in dem Teilprojekt ein besonderer Stellenwert beigemessen wird, das als einziges auf selbst erhobenen Daten basiert. Jäger ist sicher zuzustimmen, wenn er schreibt:

"Sehr früh ist uns auch bewußt geworden, daß die Erfahrungen, die wir während unserer mehr als zweijährigen Arbeit gemacht haben, selbst zum zentralen Gegenstand der Untersuchung werden würden und daher in unserem Bericht zu thematisieren seien. Denn sie illustrieren in mancher Hinsicht unmittelbar das, was wir über die analysierten Einzelfälle in Erfahrung bringen konnten, die gesellschaftlichen Kommunikationsstörungen und Zerstörungen, die sich auf terroristische Aktivitäten wie auch die Reaktionen auf sie ausgewirkt haben.« (2/125)

Die Schwierigkeiten mit der Bürokratie, mit den potentiellen Interviewpartnern (inhaftierte Terroristen und deren Angehörige) und den eigenen Kollegen führen Jäger zu der Einschätzung, mit der Terrorismusforschung "zwischen die Fronten zu geraten«. Die detaillierte Schilderung der auf allen Ebenen mit dieser Forschung verbundenen Probleme bleibt allerdings insofern folgenlos, als sich Jäger dann schließlich auf die »Perspektive einer um objektive Erkenntnis bemühten Forschung " beruft. Diese Bestimmung mutet eigenartig an, als nach der meines Erachtens sehr offenen und realistischen Schilderung der vorhandenen Restriktionen die Daten, die letztendlich in die Analyse einfließen, kaum ausreichend Informationen enthalten, um Aussagen den Status empirisch-wissenschaftlich zu verleihen. Jäger bleibt auch entsprechend vage: "Entwicklungsstrukturen « und "Bedingungsfelder" werden zu "provisorischen Generalisierungen« verarbeitet. Gerade wenn wie hier ein komplexer theoretischer Bezugsrahmen zugrunde gelegt wird, der zwar nicht unbedingt eine hohe Fallzahl, aber doch eine bestimmte Zahl sehr umfassend dokumentierter Einzelfälle voraussetzt, scheinen auf der Jäger zur Verfügung stehenden Datenbasis wissenschaftlich begründete Aussagen nicht möglich. Er bleibt sodann in seiner Darstellung auch anekdotisch. Schlußfolgerungen sind entsprechend allgemein gehalten: "... ist doch davon auszugehen, daß Belastungen und konflikthafte Erfahrungen in der Frühphase ... für den weiteren Verlauf nicht ohne Bedeutung blei- 
ben. "Die mit der datenbedingten Undifferenziertheit und der durch das theoretische Vorverständnis gestützten individualisierenden Perspektive einhergehende Gefahr ist die Kollektivstigmatisierung. Leben in Wohngemeinschaften oder Ausstieg aus »bürgerlichen Lebensformen «, verstanden als individuelle Prozesse, erscheinen als Vorlaufstation auf dem Weg in den Terrorismus. Die Strategie, alles auf das Individuum zu projizieren, ist ein übliches Verfahren beim Versuch, abweichendes Verhalten zu erklären. Die Suche nach individuellen Motiven ist dabei so trivial, wie sie erfolgreich ist. Natürlich lassen sich immer bei Personen in exponierter Position "Aufwertungsbedürfnisse « (2/152) diagnostizieren, die in wie auch immer abgemilderter Form zum Verhalten dieser Personen in Beziehung gesetzt werden können. Die Karriere von Politikern ließe sich mit einem ähnlichen Motivvokabular beschreiben, wie es hier auf die von Jäger interviewten Mitglieder der RAF angewendet wird. Nur deren Lebensgeschichten sind in der Regel kein bedrohliches und damit auch kein erklärungsbedürftiges Phänomen. Mit »verinnerlichter Gruppenmoral«, abgesichert durch »rigide Kontrollmechanismen« und der »Entwicklung einer Kontrakultur « (2/ 1 57) läßt sich Polizistenverhalten bei Demonstrationseinsätzen psychologisch ebenso erklären wie terroristisches Verhalten. Der wesentliche Unterschied ist der normative Bezugsrahmen der Analyse. Der allerdings bleibt bei Jäger zwar nicht unerwähnt, aber für die Analyse selbst unbedeutsam. Nicht daß die Psychoanalyse oder ein interpretativ psychologisches Vorgehen bei einer Anwendung auf das Problem des Terrorismus keine Ergebnisse erzeugen würde, die Frage ist jedoch, ist es angemessen, in dieser Weise sich als psychoanaly tisch orientierter Forscher an einem Projekt über Terrorismus zu beteiligen? Es erscheint fragwürdig, in diesem Zusammenhang (mit der beschränkten Datenbasis und unter den Bedingungen politischer Polarisierung) sich dem Phänomen Terrorismus in dieser Art psychologisch zu nähern.

Sind Jägers Rekonstruktionsversuche zwar problematisch, aber immer noch als sozialwissenschaftlich orientierte Interpretationen zu bezeichnen, so driftet Lorenz Böllinger in seinem Beitrag "Die Entwicklung zu terroristischem Handeln als psychosozialer Prozeß « ins sozialwissenschaftliche Abseits: Die emotionale Befindlichkeit des Analytikers wird zum unmittelbaren Evidenzkriterium erhoben. Er geht aus von der bekannten Tatsache, daß menschliche Kommunikation auf zwei Ebenen stattfindet:

"Einer offenen, bewußten und direkt beobachtbaren und einer eher verdeckten, unbewußten, nur mittelbar erschließbaren ... Deshalb sind die Gefühlsreaktionen des Interviewers und ihre Bewußtwerdung in der geschulten Selbstwahrnehmung ein äußerst wichtiges, manchmal das einzige Erkenntnisinstrument. Die Zuverlässigkeit dieses , Erfühlens $<$ verdeckter Inhalte bemißt sich lediglich an dem Maß subjektiver Evidenz.« $(2 / 176)$

Böllinger versucht, anhand von Falldarstellungen und Interpretationen die Bedeutung von »Urmißtrauen «, "Schuldgefühl « und anderer auf frühkindliche Erlebnisse bezogener Konzepte die Biographie von Terroristen zu rekonstruieren. Bemerkenswert ist, wie er dabei immer Anknüpfungspunkte für sein psychoanaly tisches Erklärungsschema findet. J. Haley hat die Technik des Psychoanalytikers als die "art of one-up-man-ship «, die Kunst, Herr über die Situation zu bleiben, beschrieben.? Nach exakt diesem Muster scheinen Böllingers Gespräche mit den Inhaftierten verlaufen zu sein:

"Erneut folgt eine lange Sentenz von theoretischen Herleitungen, die mir das Gefühl des Gequältseins verursacht. Intuitiv vermeide ich wiederum die Ebene des intellektuellen sSchlag-

7 Vgl. J. Haley, The Powertactics of Jesus Christ, New York 1969, S. 1 I ff. 

den, in dem es um den emotionalen Punktsieg und nicht um die Inhalte geht. « (2/209)

Kein frühkindliches Trauma, kein weiteres Gespräch, keine Ansatzpunkte für psychologische Interpretationen in diesem Fall? - Nein: Böllinger rekurriert auf seine Gefühle:

"Im Gespräch durchgängig erlebbar und durch Gegenübertragungsgefühle von Unterwerfung und Kränkung entschlüsselbar ist mir der von E. konstellierte Machtanspruch. Dabei ist eine sadistische Färbung spürbar, das beständige Eindringen und von mir als quälend erlebte Repetieren abgehobener Theorien." (2/210)

Am Ende ist ein von Jäger und Böllinger gemeinsam verfaßter Thesenkatalog angefügt. Die dort aufgestellten Thesen zur weiteren Diskussion hätten entweder auch ohne die vorangestellte Analyse formuliert werden können oder sind in ihrer Allgemeinheit für eine Diskussion ungeeignet. Insgesamt zeigt sich an den Beiträgen von Jäger und Böllinger, daß ein psychologisch verstehend vorgehendes Verfahren unter den konkreten Bedingungen dieser Forschung entweder in Allgemeinheiten verhaftet bleiben muß oder aber, wie im Falle Böllingers, zu einer falschen Unmittelbarkeit gefühlshafter Verfügung über Untersuchungsobjekte degeneriert.

\section{Band 3: Gruppenprozesse}

Zum Schluß noch einige Anmerkungen zum dritten und bisher letzten Band der Analysen zum Terrorismus. Dieser Band ist der umfangreichste von allen dreien. Er enthält verschiedene Fallstudien. Von Dieter Claessens und Karin de Ahna eine Studie über »Das Milieu der Westberliner 'Szene und die >Bewegung 2. Juni«. Eine Untersuchung von Wanda von Baeyer-Katte über "Das s Sozialistische Patientenkollektiv in Heidelberg" sowie eine Studie über die "Sozialen Bedingungen terroristischen Handelns. Das Beispiel der >Baader-Meinhof-Gruppe (RAF) « von Friedhelm Neidhardt. Der zweite Teil des Bandes ist überschrieben: »Vergleichende Gruppenanalysen " und umfaßt drei Beiträge: "Analysen von Strukturen terroristischer Gruppierungen « von Jo Groebel und Hubert Feger, "Linker und rechter Terrorismus. Erscheinungsformen und Handlungspotentiale im Gruppenvergleich « von Friedhelm Neidhardt und »Wege zum Ausstieg. Fördernde und hemmende Bedingungen« von Karin de Ahna.

Wir werden hier zum Abschluß etwas ausführlicher auf den Beitrag von Claessens und de Ahna eingehen, da hier die wohl umfassendste Perspektive entwickelt wird. Vor dem Hintergrund der bisher vorgestellten Forschungen könnte die Analyse von Claessens und de Ahna als Vorbild für die Terrorismusforschung bezeichnet werden. Hier wird zum einen aus einer historischen Perspektive argumentiert und zum anderen bedient sich die Analyse der »Sprache des Gegenstands", d. h. es wird dem Gegenstand kein abstrakt individualisierendes oder pathologisierendes kognitives System übergestülpt. Durch die Einbeziehung verschiedener Informationsquellen und die parallele Berücksichtigung individueller, sozialer und historischer Bedingungen in ihrem Wechselspiel gelingt es hier, die Dialektik der Entwicklung des Terrorismus am Beispiel der »Bewegung 2. Juni« zu erhellen; die konkreten, für die "Biographie» dieser Bewegung wichtigen Ereignisse werden vorgestellt, und durch die detaillierte ethnographische Schilderung erklärt sich der Terrorismus hier quasi von selbst, ohne die Krücken schlechter ätiologischer Theorien. Eine zentrale, für die Entwicklung terroristischer Gruppen verantwortliche Entwicklung sehen Claessens und de Ahna im Zerfall der Apo, eine andere wichtige Entwicklung ist die 
Eskalation der Gewalt im Rahmen der Studentenunruhen. Die Studie versucht im Ansatz den Weg einer gesellschaftlichen Selbstreflexion zu beschreiten und den Terrorismus nicht als individualpathologisches Phänomen von vornherein auszugrenzen. Beispielhaft für den Rückbezug der Analyse auf den historisch-gesellschaftlichen Status quo der Bundesrepublik sind Passagen wie die folgende:

"Die Diffusität, mit der in Deutschland politische Kategorien gehandhabt werden, hat in der deutschen Geschichte dazu geführt, daß die Bevölkerung wohl nie in der Polizei ein nur ausführendes d. h. exekutives Organ einer Staatsgewalt gesehen hat, die im Rahmen der Gewaltenteilung grundgesetzmäßig Eigenhemmungen für ihr Tun eingebaut hatte. Wenn man es überspitzt ausdrücken will, bedeutete in einer Bevölkerung, in der jede Abweichung mit großer Skepsis betrachtet wurde - und in Berlin hatte sich dieser Provinzialismus wie oben angeführt mehr und mehr ausgebreitet - das Auftreten von >Abweichung den Verdacht, jemand vor sich zu haben, der grundsätzlich `verdächtig`war. Unter solchen Verhältnissen ist die Bereitschaft, einen Angeschuldigten bereits als smehr als verdächtigs und in gewisser Weise damit auch schon als überführt anzusehen, groß. Setzte sich die Polizei mit jemandem auseinander, so konnte man damit rechnen, daß erhebliche Teile, besonders der älteren Bevölkerung, grundsätzlich auf der Seite der Polizei sein würden. Dieses in Deutschland schon oft mißbrauchte Staatsvertrauen führte insgesamt zu einem Verhalten, das durch die Kette: Verdacht, Fahndung, Festnahme, Verurteilung gekennzeichnet ist. Im Hinblick auf dieses gefährliche Syndrom gab es sicher große Kreise der Bevölkerung, für die ein von der Polizei Festgenommener bereits praktisch ein - und zwar zu recht - Verurteilter war. Diese im Nationalsozialismus zur Selbstverständlichkeit erhobene Haltung, wobei parapolizeiliche Formationen wie SA oder SS einbezogen waren, erfuhr selbstverständlich nach 1945 eine gewisse Abschwächung. In die Polizeiführung rückte aber eine Generation ein, die demselben Syndrom verhaftet war und es mit bestem Gewissen für die neue Republik respektive das neue demokratische Westberlin in Anspruch nahm." $(3 / 51)$

Breiten Raum nimmt in Claessens und de Ahnas Darstellung die Vorgeschichte der Studentenbewegung ein. Die als Abreißen der gemeinsamen Erfahrung apostrophierte Entwicklung, die zum offenen Ausbruch des Konflikts zwischen Studentenschaft und Universitätsestablishment führte, wird hier verständlich beschrieben. Die sich anschließenden Ereignisse werden erklärt als Eskalation, an der die Staatsorgane einen erheblichen Anteil hatten. Die Verletzung von Spielregeln durch die Polizei spielt dabei eine herausragende Rolle: »Der Grundsatz der Verhältnismäßigkeit der Mittel wurde mehr und mehr mißachtet.« (3/53) Vor dem Hintergrund einer durch weite Teile der Presse unterstützten Mentalität konnten harte Reaktionen auf studentischen Protest mit breiter Zustimmung rechnen. Zitate aus Zeitungsartikeln, die Claessens und de Ahna heranziehen, dokumentieren Reaktionen der veröffentlichten Meinung. "Wir haben es hier mit einer akademischen Variante des Gammlertums zu tun", schreibt M. Walden 1967 in der "Welt «; im selben Artikel ist die Rede von »vorsätzlicher geistiger Ungewaschenheit « und empfohlen wird, auf einen groben Klotz einen groben Keil zu setzen. (vgl. 3/63)

Rekonstruiert man die Entwicklung der Ereignisse mit der hier zugrundegelegten Genauigkeit, so stößt man auch auf den rationalen Kern von letztlich im Lauf der Eskalation des Terrorismus ins Irrationale abgedrifteten Schlagworten wie dem des "Faschismusvorwurfs". Wie solche Schlagworte entstehen konnten, wird verständlich im Angesicht der Ereignisse nach der Erschießung Benno Ohnesorgs. Die Haltung der politisch Verantwortlichen und deren Vorschläge (etwa ein Demonstrationsverbot) ließen damals - auch im Ausland - die Erinnerung an die Zeit vor 1945 wiedererstehen:

"In das Zielfeld von Befürchtungen rückten Faschismusverdacht und allgemein die Gefährdung von Demokratie und Freiheit. Zu ersterem werden Erinnerungen an das $>$ Dritte Reiche aktualisiert und versichert, daß (von der britischen Bevölkerung) mit größter Besorgnis alle Entwicklungen beobachtet werden, die in Richtung eines neuen deutschen Polizeistaates gehn und alles getan werden müsse, um das zu verhindern. Befürchtet wird, daß >der Geist von damals bzw. 
der Ungeist ‘ neu erstanden sei, denn >wie damals liebt man es, erst einmal auf die Geistigen loszuprügeln, um damit den Geist jeden Widerspruchs einzuschüchtern<.

Bereits 1933 sei das Eintreten für demokratische Grundrechte mit 'Schutzhaftlager bestraft worden; die Planung derartiger einfacher, Ordnungsmaßnahmen von gewissen politischen Kreisen in Deutschland sei wieder zu befürchten.« $(3 / 75)$

Die Ereignisse des 2. Juni 1967 hatten eine wichtige Katalysatorfunktion allerdings weniger für die Entstehung terroristischer Aktivitäten als für die Entwicklung der APO. Sie ermöglichten eine radikalere Politisierung der Studentenschaft und förderten eine gemeinsame Orientierung, die sich als Ziel die Veränderung gesellschaftlicher Verhältnisse in der BRD mit der Hoffnung auf mehr Demokratie setzte:

"Es ist wichtig, sich dessen zu erinnern, daß es damals nicht zu ,Vergeltungsanschlägen oder aggressiv-destruktiven Entladungen von Empörung, Wut und Erbitterung kam wie knapp ein Jahr später nach dem Attentat auf Rudi Dutschke oder in den 7oer Jahren, wo das fast zur Gewohnheit wird als Antwort auf vergleichsweise geringe Anlässe. (Allerdings kann dann auch nicht mehr von >der/ studentischen Bewegung gesprochen werden.) " (3/80)

Claessens und de Ahna gelingt es, das Zusammenspiel subkulturell-sozialpsychologischer und objektiver Entwicklungen zu erfassen. Eingefügte Hinweise auf die Bedeutung von Einflüssen, in denen sich biographische und historische Aspekte vermischen (etwa die Identifikationsproblematik der Nachkriegsgeneration) sind sinnvoll, wenn sie in Bezug gesetzt werden zum gesellschaftlichen Kontext, in dem sie auftauchen. RAF und Bewegung 2. Juni, die skandalisierte Spitze des Eisbergs, auf die sich die meisten der anderen Teilprojekte mehr oder weniger beschränkt beziehen und die dort vielfach bloß geschichtslose Monstren sind, erscheinen hier als - allerdings fatales - Produkt einer rational rekonstruierbaren Entwicklung, als Folge der Unfähigkeit einer Gesellschaft, sich politischer Kritik zu stellen und anders als repressiv darauf zu reagieren.

Reinhard Kreissl 